\title{
Evaluation of risk factors associated with pancreatic adenocarcinoma in Black Sea region, Turkey
}

\author{
Murat Derebey $^{1}$ Kagan Karabulut ${ }^{1} \cdot$ Saim Savas Yuruker $^{1} \cdot$ Ilhan Karabicak $^{\mathbf{2}}$ - Necati Ozen ${ }^{\mathbf{1}}$ \\ ${ }^{1}$ Department of General Surgery, Faculty of Medicine, Ondokuz Mayis University, Samsun, Turkey \\ ${ }^{2}$ Department of General Surgery, VM Medical Park Hospital, Samsun, Turkey
}

\section{ABSTRACT}

\begin{abstract}
Aim: To evaluate the risk factors in patients with pancreatic adenocarcinoma in Turkey's Black Sea region and to determine groups at high risk for pancreatic cancer.

Methods: 106 newly diagnosed pancreatic adenocarcinoma patients living in Black Sea region of Turkey who applied to our clinic between January 2015 and December 2016 were included in this study. In the same period a control group was formed with 92 patients of similar age. Both groups were asked to fill out a form that questioned the pancreatic cancer risk factors. Data were analyzed.

Results: Pancreatic cancer risk was 3.5 times higher in people over 65 years of age. Patients with deficient level of serum vitamin D level $(<20 \mathrm{ng} / \mathrm{ml})$ had 10.2 times more risk of pancreatic cancer than patients with normal level of Vitamin D ( $\geq 30 \mathrm{ng} / \mathrm{ml}$ ). While newly diagnosed type 2 diabetics had 19.5 times higher risk of pancreatic cancer, long term type 2 diabetics had 1.2 times higher risk than normal individuals. Those with pancreatic cancer in family had 4.3 times higher risk than those who did not. It was observed that people with rhesus ( $\mathrm{Rh}$ ) antigen negative blood group has $70 \%$ less risk of pancreatic cancer.
\end{abstract}

Conclusion: Determination of pancreatic cancer risk factors, organization of imaging and screening programs for high-risk people can provide early diagnosis of the disease and prolong survival.

Keywords: Pancreatic adenocarcinoma, risk factors, pancreatic neoplasms, etiology, epidemiology.

Dr. Murat Derebey,

Department of General Surgery, Ondokuz Mayls

University, Faculty of Medicine, Samsun, Turkey

E-mail: mderebey@gmail.com

Received: 2020-10-05 / Revised: 2020-10-20

Accepted: 2020-10-29 / Published online: 2021-01-01

\section{Introduction}

Although pancreatic cancer is the 11th most common cancer type, it ranks as the third leading cause of cancer-related death in the USA [2]. Pancreatic cancer is predicted to be the second most common cause of cancerrelated deaths in coming 20 years [3]. In a study conducted by Parkin et al. in 2002, pancreatic cancer accounts for $3 \%$ of all cancer types worldwide, however, it is among the top in cancer-related deaths due to its poor diagnosis [1]. In many patients, when pancreatic cancer is detected, the disease is advanced and the tumor is not operable. Median survival rate is less than a year, and mortality rate is approximately $99 \%$. Only less than $10 \%$ of pancreatic tumors can be detected at an early phase and resected. Usually the disease starts to develop two years before its diagnosis [4]. Most patients initially have a non-specific pain in epigastrium. As the disease develops in time, the pain spreads over to the back, upper thoracic and lumbar region. Upon progress of the disease, most patients consult doctors with complaints of pain, jaundice, and 
loss of weight. It is often the case that when patient seeks medical advice with these complaints, the disease has already reached the advanced stage [1-5].

There are numerous studies conducted to identify causes of pancreatic cancer. In etiology, environmental and genetic causes are considered to play a joint role. Risk factors are divided in two as changeable and unchangeable risk factors. Unchangeable risk factors are age, gender, race, genetic factors, family history, atopy/allergy history, blood groups, surgery history, diabetes and chronic pancreatitis. Changeable risk factors, on the other hand, can be listed as smoking, obesity, use of alcohol, dietary, socio-economic conditions, infections, levels of vitamin D and folic acid. Analysis of risk factors and identification of factors that significantly increases the risk are of high importance [1-10]. Identifying populations of high-risk, establishing imaging and screening programs for these groups can support early diagnosis of the disease and increase survival period. Therefore, in this study, we aimed to identify high-risk groups together with risk factors in patients with pancreatic adenocarcinoma in Turkey's Black Sea region.

\section{Materials and Methods}

Ethical approval for this study was obtained from the local ethics committee (IRB approval number 144/2015). Necessary permissions were obtained from the hospital and families. All procedures performed in studies involving human participants were in accordance with the ethical standards of the institutional and/or national research committee and with the 1964 Helsinki declaration and its later amendments or comparable ethical standards.

106 newly diagnosed pancreatic adenocarcinoma patients living in Black Sea Region of Turkey who applied to our clinic between January 2015 and December 2016 were included in this study. In the same period, a control group was also formed with 92 patients of similar age group who visited our clinic for another disease and had not been diagnosed with cancer. Both groups were asked to fill out a form that questioned the risk factors. The factors to be investigated in this study, conducted as a case-controlled and crosssectional study, are defined as follows: age $(\leq 50,51-65,>65)$; gender (female, male); family history of pancreatic cancer (yes, no); atopy/allergy (yes, no); blood type (A,B,O,AB; rhesus (Rh) antigen negative, $\mathrm{Rh}$ antigen positive); cholecystectomy (yes, no); gastrectomy (yes, no); type 2 diabetes (yes, no); duration of diabetes - newly diagnosed type 2 diabetes ( $<2$ years), long term type 2 diabetes ( $\geq 2$ years); smoking (never smoked, still smoking or stopped smoking); body mass index (BMI) normal weight (18.5-24.99), pre-obesity (25-29.99), obesity ( $\geq 30)$; alcohol consumption (yes, no); place of residence (rural, urban); helicobacter pylori history (yes, no); level of vitamin D normal $(>30,80 \mathrm{ng} / \mathrm{ml})$, insufficient $(20, \leq 30 \mathrm{ng} / \mathrm{ml})$, deficient $(<20 \mathrm{ng} / \mathrm{ml})$; level of folic acid low $(<4.6 \mathrm{ng} / \mathrm{ml})$, normal (4.6-18.7 $\mathrm{ng} / \mathrm{ml})$, high ( $>18.7 \mathrm{ng} / \mathrm{ml})$.

\section{Statistical analysis}

The collected data were analyzed with IBM SPSS V23. In the evaluation of the study data, besides the descriptive statistical methods (mean, standard deviation), in the comparison of qualitative data; Pearson's chi-squared test were used. The $p$-value $<0.05$ was significantly considered. Independent Sample T-test was applied in the analysis of the significance of the difference between the averages obtained.

\section{Results}

There was no significant difference across groups concerning age and gender $(p>0.05)$ 
(see Table 1). Pancreatic cancer risk was found 3.5 times higher in patients over 65 years of age compared to the control group $(p<0.05)$. Gender was not associated with increased pancreatic cancer risk $(p>0.05)$. The risk of pancreatic cancer was observed 3.4 times higher in people with insufficient serum vitamin D level. For people with deficient level of serum vitamin $\mathrm{D}$, the risk increased 10.2 times more $(p<0.05)$. When non-smokers were taken as reference, there was no increase in the risk of pancreatic cancer in individuals who quit smoking or who are smoking $(p>0.05)$. The risk of pancreatic cancer was detected to increase 1.2 -fold in diabetic patients $(p<0.05)$. When diabetic patients were classified as newly diagnosed type 2 diabetes ( $<2$ years) and longterm type 2 diabetes ( $\geq 2$ years), the risk of pancreatic cancer increased by 19.5 -fold in newly diagnosed type 2 diabetic patients $(p<0.05)$. There was no association between BMI, alcohol use, chronic pancreatitis and pancreatic cancer risk $(p>0.05)$. Compared to the control group, the risk of pancreatic cancer was observed to increase 4.3 -fold in patients with pancreatic cancer history in the family $(p<0.05)$.
There was no significant difference in terms of pancreatic cancer risk between those living in urban areas and rural areas $(p>0.05)$. Similarly, we observed no relation between the level of serum folic acid, distribution of $\mathrm{ABO}$ blood types, helicobacter pylori, atopy/allergy, gastrectomy, cholecystectomy, and the risk of pancreatic cancer $(p>0.05)$. However, the risk of pancreatic cancer was found less patients with $\mathrm{Rh}$ antigen negative blood group $(p<0.05)$ (See table 2).

There was no significant difference across groups concerning age and gender $(p>0.05)$ (See Table 1). Pancreatic cancer risk was found 3.5 times higher in patients over 65 years of age compared to the control group $(p<0.05)$. Gender was not associated with increased pancreatic cancer risk $(p>0.05)$. The risk of pancreatic cancer was observed 3.4 times higher in people with insufficient serum vitamin D level. For people with deficient level of serum vitamin $\mathrm{D}$, the risk increased 10.2 times more $(p<0.05)$. When non-smokers were taken as reference, there was no increase in the risk of pancreatic cancer in individuals who quit smoking or who are smoking $(p>0.05)$. The risk of pancreatic cancer was detected to increase

Table 1. Age and gender distribution.

\begin{tabular}{|c|l|l|l|}
\hline Parameters & Pancreatic Cancer & Control Group & P value \\
\hline Average age (Years) & $65 \pm 11$ & $62 \pm 13$ & \multirow{2}{*}{0.207} \\
\hline $\begin{array}{c}\text { Age groups } \leq \mathbf{5 0} \\
\mathbf{5 1 - 6 5}\end{array}$ & $11(10.4 \%)$ & $19(20.7 \%)$ & \multirow{2}{*}{0.052} \\
\cline { 2 - 3 } $\mathbf{6 5 5} \quad$ Male & $62(58.5 \%)$ & $33(35.9 \%)$ & \\
\cline { 2 - 3 } Female & $63(59.4 \%)$ & $52(56.5 \%)$ & \multirow{2}{*}{0.679} \\
\cline { 2 - 4 } & $43(40.6 \%)$ & $40(43.5 \%)$ & \\
\hline
\end{tabular}


Table 2. Comparison of pancreatic cancer risk factors between groups.

\begin{tabular}{|c|c|c|c|c|}
\hline Parameters & $\begin{array}{l}\text { Pancreatic Cancer } \\
\text { Group }\end{array}$ & Control Group & OR & $P$ \\
\hline \multicolumn{5}{|l|}{ Age groups } \\
\hline \multirow{3}{*}{$\begin{array}{l}\leq 50 \\
51-65 \\
>65\end{array}$} & $11(10.4 \%)$ & $19(20.7 \%)$ & Reference & \multirow{3}{*}{0.014} \\
\hline & $33(31.1 \%)$ & $33(35.9 \%)$ & $1.3(0.4-4.1)$ & \\
\hline & $62(58.5 \%)$ & $40(43.5 \%)$ & $3.5(1.2-10.2)$ & \\
\hline \multicolumn{5}{|l|}{ Gender } \\
\hline \multirow{2}{*}{$\begin{array}{l}\text { Male } \\
\text { Female }\end{array}$} & $63(59.4 \%)$ & $52(56.5 \%)$ & Reference & \multirow{2}{*}{0.616} \\
\hline & $43(40.6 \%)$ & $40(43.5 \%)$ & $0.9(0.4-2.2)$ & \\
\hline \multicolumn{5}{|l|}{ Serum vitamin D level (ng/ml) } \\
\hline \multirow{3}{*}{$\begin{array}{l}\text { Normal }(>30,80) \\
\text { Insufficient }(20, \leq 30) \\
\text { Deficient }(<20)\end{array}$} & $3(2.8 \%)$ & $10(10.9 \%)$ & Reference & \multirow{3}{*}{0.001} \\
\hline & $6(5.7 \%)$ & $15(16.3 \%)$ & $3.4(0.5-25.4)$ & \\
\hline & $97(91.5 \%)$ & $67(72.8 \%)$ & $10.2(2-52.6)$ & \\
\hline \multicolumn{5}{|l|}{ Smoking } \\
\hline \multirow{2}{*}{$\begin{array}{l}\text { Never smoked } \\
\text { Still smoking or smoked before/quit smoking }\end{array}$} & $51(48.1 \%)$ & $52(56.5 \%)$ & Reference & \multirow{2}{*}{0.242} \\
\hline & $55(51.9 \%)$ & $40(43.5 \%)$ & $1.6(0.7-3.8)$ & \\
\hline \multicolumn{5}{|l|}{ Body mass index $\left(\mathrm{kg} / \mathrm{m}^{2}\right)$} \\
\hline \multirow{3}{*}{$\begin{array}{l}\text { Normal }(18.5-24.99) \\
\text { Pre-obesity }(25-29.99) \\
\text { Obesity }(\geq 30)\end{array}$} & $22(20.8 \%)$ & $28(30.4 \%)$ & Reference & \multirow{3}{*}{0.757} \\
\hline & $44(41.5 \%)$ & $32(34.8 \%)$ & $1.7(0.7-4.3)$ & \\
\hline & $40(37.7 \%)$ & $32(34.8 \%)$ & $1.3(0.5-3.6)$ & \\
\hline \multicolumn{5}{|l|}{ Type 2 diabetes } \\
\hline No & $65(61.3 \%)$ & $73(79.3 \%)$ & Reference & \\
\hline Yes & $41(38.7 \%)$ & $19(20.7 \%)$ & $1.2(0.4-3.1)$ & 0.001 \\
\hline Duration of type 2 diabetes (years) & & & & \\
\hline No & $65(61.3 \%)$ & $73(79.3 \%)$ & Reference & \\
\hline Newly diagnosed type 2 diabetes ( $<2$ years) & $23(21.7 \%)$ & $2(2.2 \%)$ & $19.5(3-127.3)$ & 0.003 \\
\hline Long term type 2 diabetes ( $\geq 2$ years) & $18(17.0 \%)$ & $17(18.5 \%)$ & $1.2(0.6-2.5)$ & \\
\hline Family history of pancreatic cancer & & & & \\
\hline No & $96(90.6 \%)$ & $89(96.7 \%)$ & Reference & \\
\hline Yes & $10(9.4 \%)$ & $3(3.3 \%)$ & $4.3(0.8-22.3)$ & 0.041 \\
\hline Alcohol consumption & & & & \\
\hline No & $88(83.0 \%)$ & $77(83.7 \%)$ & Reference & \\
\hline Yes & $18(17.0 \%)$ & $15(16.3 \%)$ & $0.9(0.3-2.6)$ & 0.196 \\
\hline Place of residence & & & & \\
\hline Rural & $50(47.2 \%)$ & $27(29.3 \%)$ & $2(0.9-4.3)$ & \\
\hline Urban & $56(52.8 \%)$ & $65(70.7 \%)$ & Reference & 0.054 \\
\hline Chronic pancreatitis history & & & & \\
\hline No & $104(98.1 \%)$ & $91(98.9 \%)$ & Reference & \\
\hline Yes & $2(1.9 \%)$ & $1(1.1 \%)$ & $0.7(0.1-32.4)$ & 0.814 \\
\hline Helicobacter pylori history & & & & \\
\hline No & $93(87.7 \%)$ & $85(92.4 \%)$ & Reference & \\
\hline Yes & $13(12.3 \%)$ & $7(7.6 \%)$ & $1.5(0.5-5.3)$ & 0.310 \\
\hline Serum folic acid level (ng/ml) & & & & \\
\hline Low $(0,<4.6)$ & $9(8.5 \%)$ & $18(19.6 \%)$ & Reference & \\
\hline Normal (4.6-18.7) & $91(85.8 \%)$ & $69(75.0 \%)$ & $3.7(1.2-11.5)$ & 0.099 \\
\hline High (>18.7) & $6(5.7 \%)$ & $5(5.4 \%)$ & $2.8(0.5-15.1)$ & \\
\hline Blood groups & & & & \\
\hline $\mathrm{O}$ & $30(28.3 \%)$ & $32(34.8 \%)$ & Reference & \\
\hline A & $56(52.8 \%)$ & $36(39.1 \%)$ & $1.5(0.7-3.4)$ & \\
\hline B & $14(13.2 \%)$ & $19(20.7 \%)$ & $0.6(0.2-1.8)$ & 0.835 \\
\hline $\mathrm{AB}$ & $6(5.7 \%)$ & $5(5.4 \%)$ & $1.1(0.2-5.4)$ & \\
\hline Rh status & & & & \\
\hline Rh antigen negative & $91(85.8 \%)$ & $69(75.0 \%)$ & Reference & 0.006 \\
\hline $\mathrm{Rh}$ antigen positive & $15(14.2 \%)$ & $23(25.0 \%)$ & $0.3(0.1-0.8)$ & 0.006 \\
\hline Atopy/allergy & & & & \\
\hline No & $99(93.4 \%)$ & $82(89.1 \%)$ & Reference & 0.453 \\
\hline Yes & $7(6.6 \%)$ & $10(10.9 \%)$ & $0.6(0.1-2.3)$ & 0.453 \\
\hline Cholecystectomy & & & & \\
\hline No & $91(85.8 \%)$ & $83(90.2 \%)$ & Reference & \\
\hline Yes & $15(14.2 \%)$ & $9(9.8 \%)$ & $1.6(0.5-5.3)$ & 0.406 \\
\hline Gastrectomy & & & & \\
\hline No & $102(96.2 \%)$ & $90(97.8 \%)$ & Reference & \\
\hline Yes & $4(3.8 \%)$ & $2(2.2 \%)$ & $1.1(0.2-8.2)$ & 0.926 \\
\hline
\end{tabular}


1.2 -fold in diabetic patients $(p<0.05)$. When diabetic patients were classified as newly diagnosed type 2 diabetes ( $<2$ years) and longterm type 2 diabetes ( $\geq 2$ years), the risk of pancreatic cancer increased by 19.5 -fold in newly diagnosed type 2 diabetic patients $(p<0.05)$. There was no association between BMI, alcohol use, chronic pancreatitis and pancreatic cancer risk $(p>0.05)$. Compared to the control group, the risk of pancreatic cancer was observed to increase 4.3 -fold in patients with pancreatic cancer history in the family $(p<0.05)$. There was no significant difference in terms of pancreatic cancer risk between those living in urban areas and rural areas $(p>0.05)$. Similarly, we observed no relation between the level of serum folic acid, distribution of $\mathrm{ABO}$ blood types, helicobacter pylori, atopy/allergy, gastrectomy, cholecystectomy, and the risk of pancreatic cancer $(p>0.05)$. However, the risk of pancreatic cancer was found less patients with $\mathrm{Rh}$ antigen negative blood group $(p<0.05)$ (see Table 2).

\section{Discussion}

In the United States, $10 \%$ of pancreatic cancers are under the age of 50. It has been reported that the risk of cancer increases with age [5-7]. When people aged 50 and below are referenced, the risk of pancreatic cancer has increased 3.5fold for those who are older than $65(p<0.05)$. Many studies on pancreatic cancer have not revealed a significant difference between genders $[8,9]$. In our study, gender was not correlated to pancreatic cancer risk $(p>0.05)$. The best indicator of vitamin D level in serum is 25-hydroxyvitamin D3. This level is determined by dietary vitamin $\mathrm{D}$ and sun exposure. It is stated that serum vitamin D level may vary depending on age, race, BMI and diseases. Experimental studies suggest that the risk of pancreatic cancer may be reduced if the optimal level of serum vitamin D is achieved, depending on the diet or sun rays. However, clinical studies are inadequate; there are some conflicting data available, too. In one of these, it has been suggested that there may be an increased risk of pancreatic cancer with increased serum 25-hydroxyvitamin D3 levels. However, at the end of the study, the difference was considered to stem from the difference in patient-control group analysis of the studied population or the difference in the method used to measure the level of 25-hydroxyvitamin D3 [10]. In an experimental study conducted by Kasiappan et al. [11] a group of mice with tumors were given a high-fat diet for 30 days; another group with a tumor was given a highfat diet and the vitamin D analogue seacalcitol (EB1089). When the two groups were compared, tumor growth was more than $50 \%$ in mice that did not receive the vitamin $\mathrm{D}$ analogue seacalcitol. In our study, the risk of pancreatic cancer increased by 10.2 -fold in people with deficient serum D level compared to individuals with normal vitamin D level $(p<0.05)$. Pre-clinical studies contain data which indicates that cancer risk will decrease with vitamin D support. Clinical studies cannot adequately express this. This relationship is maybe really weak; perhaps it may be present in certain cancers.

Smoking is the most important known risk factor associated with pancreatic cancer. In a study, the risk of pancreatic cancer was found to be two times higher in smokers than in nonsmokers. It was stated that this risk would increase in direct proportion with the amount of cigarettes used and the duration of time smoked [12]. According to our data, there was no correlation between smoking and the risk of pancreatic cancer $(p>0.05)$. Based on American Cancer Society studies, those with BMI $\geq 30$ had a 2.08 times greater risk of pancreatic 
cancer than those with BMI $<25$ [13]. In the data obtained, there was no correlation BMI and pancreatic cancer risk $(p>0.05)$. Diabetes is a risk factor for pancreatic cancer; it can also be a sign of the emergence of pancreatic cancer or a condition that may occur after surgery. In a meta-analysis of 20 studies conducted by Everhart and Wright in 1995, the risk of pancreatic cancer in diabetic individuals was found to be 2 times more than in non-diabetic [1]. In the data obtained, the risk of pancreatic cancer in diabetic patients was found 1.2 times higher than in non-diabetic patients $(p<0.05)$. In a study conducted with 1428 pancreatic cancer and 1528 healthy control groups, the rate of diabetes was $27.6 \%$ in people with pancreatic cancer, while the rate of diabetes was $9.9 \%$ in people in the control group. Of the patients who have both pancreatic cancer and diabetes, $76.2 \%$ had newly diagnosed diabetes, which was significantly higher than the control group [14]. When non-diabetic patients were referenced, the risk of pancreatic cancer increased 19.5-fold in the presence of newly diagnosed type 2 diabetes ( $<2$ years); those with long-term type 2 diabetes ( $\geq 2$ years) had a 1.2fold increased risk of pancreatic cancer $(p<0.05)$.

Familial pancreatic cancer accounts for $10 \%$ of all patients with pancreatic cancer. In a study, the risk of pancreatic cancer for people in whose immediate family (mother, father, and siblings) there are at least two with pancreatic cancer increases the risk ten times compared those with no family history [15]. According to our data, the risk of cancer increased 4.3 times in patients with a family history of pancreatic cancer $(p<0.05) .1530$ pancreatic cancer patients and 1530 healthy individuals from the Pancreatic Cancer Cohort Consortium were evaluated in a case-control study. There was no significant statistical difference between those who had $>0$ or $<5$ grams of alcohol daily and those who had $\geq 60$ grams of alcohol daily [16]. In another study, it was reported that consuming 40 grams of alcohol daily increased the risk of pancreatic cancer 1.45 times, and that it could increase up to 1.62 times [17]. The results from these studies are contradictory. In our study, there was no correlation between alcohol use and the risk of pancreatic cancer $(p>0.05)$.

Research has shown that pancreatic cancer is more common in people living in urban areas with low socioeconomic condition. According to our data, no relationship was found between the place of residence and the risk of pancreatic cancer $(p>0.05)$. Four percent of patients with chronic pancreatitis are believed to develop pancreatic cancer [18]. If a person has a history of familial pancreatitis, the risk increases 50-60 times [19]. In our study, there was no correlation between the risk of chronic pancreatitis and pancreatic cancer $(p>0.05)$. The International Agency for Research on Cancer classifies the helicobacter pylori as a proven carcinogen. In four contemporary studies conducted, it was iterated that there is a possible relationship between pancreatic cancer and helicobacter pylori, however further studies are needed [20]. According to our data, there was no correlation between helicobacter pylori and pancreatic cancer risk $(p>0.05)$. The International Agency for Research on Cancer has proposed that foods containing folate may be protective against pancreatic cancer [21]. In a study evaluating 14 prospective cohort studies that involves 319716 men and 542948 women, the relationship between folate use and pancreatic cancer was investigated. Over 7-20 years after the study, 2195 pancreatic cancers were identified. There was no correlation between folate intake and pancreatic cancer risk when the $15 \%$ quartile of the highest folate intake diet and $15 \%$ quartile of the lowest folate 
intake diet were compared [22]. In the current literature, inconsistent findings have been observed between the amount of folate in the diet and the level of serum folate and the risk of pancreatic cancer. In the data obtained, no correlation between folic acid level and pancreatic cancer risk $(p>0.05)$ was found.

In a study conducted on blood types with 224 patients who have pancreatic cancer, the risk was 1.5 times higher in individuals with blood type B than in individuals with other blood types; the risk was lower in individuals with 0 type blood [23]. In our study, there was no correlation between ABO blood types and pancreatic cancer risk $(p>0.05)$. It has been investigated whether there is any difference in pancreatic cancer risk based on Rh status or not. According to the statistical analysis, the risk of pancreatic cancer in $\mathrm{Rh}$ antigen negative people was $70 \%$ less $(p<0.05)$. Given the differences in distribution of $\mathrm{ABO}$ and $\mathrm{Rh}$ blood types of people living all over the world and current studies concerning blood types, further studies are needed to determine whether there is a link between blood types and pancreatic cancer risk. When 13 community-based studies were examined, the risk of pancreatic cancer in atopic/allergic individuals was 30\% less [24]. Studies examining the relationship between pancreatic cancer and atopy/allergy suggest that there is need for further studies. According to our data, there was no significant association between atopy/allergy and pancreatic cancer risk $(p>0.05)$. A study shows that cholecystectomy increased the risk of pancreatic cancer by $23 \%$ and gastrectomy by $50 \%[25,26]$. In our study, there was no relation between the risk of pancreatic cancer and cholecystectomy and/or gastrectomy ( $p>0.05)$. In the current literature, the relationship between cholecystectomy or gastrectomy and the risk of pancreatic cancer is not clear enough.
The limitation of this study is that it is casecontrolled and cross-sectional. Therefore, the results may suggest that vitamin D deficiency is more common in patients with pancreatic cancer, but we cannot clearly predict the increased risk of pancreatic cancer in cases of vitamin D deficiency. Smoking is a proven risk factor for pancreatic cancer, but this was not demonstrated in our study. On the other hand, a similar situation applies to the relationship between $\mathrm{Rh}$ status and pancreatic cancer risk. We cannot expressly conclude that the risk of pancreatic cancer is reduced in people with $\mathrm{Rh}$ antigen negative. A population-based follow-up study is required for more reliable results. This article may contribute to the following population-based studies.

The main problem with pancreatic cancer is that the disease cannot be detected early; when diagnosed, the disease is often at an advanced stage. Because the survival rate is low and that diagnosis cannot be made in the early stages of pancreatic cancer, primary prevention is important. Therefore, identification of risk factors and better elucidation of etiology is necessary.

\section{Conclusion}

Determination of pancreatic cancer risk factors, organization of imaging and screening programs for high-risk people can provide early diagnosis of the disease and prolong survival. Population-based studies are needed to reveal pancreatic cancer risk factors.

Funding: There is no financial support and sponsorship

Conflict of Interest: The authors declare that they have no conflict of interest.

Ethical statement: The study was conducted in accordance with the ethical approval of the 
University Ethics Committee (Date and decision number: 144/2015).

ORCID iD of the author(s)

Murat Derebey / 0000-0002-0654-846X

Kagan Karabulut / 0000-0003-4723-5360

Saim Savas Yuruker / 0000-0002-6371-337X

Ilhan Karabicak / 0000-0002-3505-9534

Necati Ozen / 0000-0002-8072-9234

Copyrights: (C) 2021@author (s).

This is an open access article distributed under the terms of the Creative Commons Attribution License (CC BY 4.0), which permits unrestricted use, distribution, and reproduction in any medium, provided the original author(s) and source are credited and that the original publication in this journal is cited, in accordance with accepted academic practice. No use, distribution or reproduction is permitted which does not comply with these terms.

\section{References}

[1]Huxley R, Moghaddam A, Berrington de Gonzalez A, et al. Type-II diabetes and pancreatic cancer: a meta-analysis of 36 studies. Br J Cancer. 2005;92(11):2076-83.

[2]Siegel RL, Miller KD, Jemal A. Cancer statistics, 2019. CA Cancer J Clin. 2019;69(1):7-34.

[3]Torre LA, Bray F, Siegel RL, et al. Global cancer statistics, 2012. CA Cancer J Clin. 2015;65(2):87-100.

[4]Kimura Y, Hirata K, Mukaiya M, et al. Hand-assisted laparoscopic pyloruspreserving pancreaticoduodenectomy for pancreas head disease. Am J Surg. 2005;189(6):734-37.

[5]Ilic M, Ilic I. Epidemiology of pancreatic cancer. World J Gastroenterol. 2016;22(44):9694-5.
[6]Lippi G, Mattiuzz C. The global burden of pancreatic cancer. Arch Med Sci. 2020;16(4):820-24.

[7]Hidalgo M, Cascinu S, Kleeff J, et al. Addressing the challanges of pancreatic cancer: future directions for improving outcomes. Pancreatology. 2015;15(1):8-18.

[8]Ferlay J, Soerjomataram I, Ervik M, et al. Cancer incidence and mortality worldwide. International Agency for Research on Cancer, 2013; http://globocan.iarc.fr 5.

[9]American Cancer Society. Cancer facts and figures 2013. Atlanta, Ga: American Cancer Society, 2013.

[10]Stolzenberg-Solomon RZ, Jacobs EJ, et al. Circulating 25-hydroxyvitamin D and risk of pancreatic cancer: Cohort Consortium Vitamin D Pooling Project of Rarer Cancers. Am J Epidemiol. 2010;172(1):81-93.

[11]Kasiappan R, Sun Y, Lungchukiet P, et al. Vitamin D suppresses leptin stimulation of cancer growth through microRNA. Cancer Res. 2014;74(21):6194-204.

[12]Iodice S, Gandini S, Maisonneuve P, et al. Langenbecks Arch Surg. 2008;393(4):53545.

[13]Calle EE, Rodriguez C, Walker-Thurmond $\mathrm{K}$, et al. Overweight, obesity, and mortality from cancer in a prospectively studied cohort of U.S. adults. N Engl J Med. 2003;348(17):1625-38.

[14]Pannala R, Leirness JB, Bamlet WR, et al. Prevalence and clinical profile of pancreatic cancer-associated diabetes mellitus. Gastroenterology. 2008;134(4):981-87.

[15]Benzel J, Fendrich V. Familial Pancreatic Cancer. Oncol Res Treat. 2018;41(10):61118.

[16]Michaud DS, Vrieling A, Jiao L, et al. Alcohol intake and pancreatic cancer: a pooled analysis from the pancreatic cancer 
cohort consortium. Cancer Causes Control. 2010;21(8):1213-5.

[17]Farrow DC, Davis S. Risk of pancreatic cancer in relation to medical history and the use tobacco, alcohol and coffee. Int $\mathrm{J}$ Cancer. 1990;45(5):816-20.

[18]Kudo Y, Kamisawa T, Anjiki $\mathrm{H}$, et al. Incidence of and risk factors for developing pancreatic cancer in patients with chronic pancreatitis. Hepatogastroenterology. 2011;58(106):609-11.

[19]Lowenfels AB, Maisonneuve P, Whitcomb DC. Risk factors for cancer in hereditary pancreatitis. International Hereditary Pancreatitis Study Group. Med Clin North AM. 2000;84(3):565-73.

[20]Bulajic M, Maisonneuve P, SchneiderBrachert W, et al. Helicobacter pylori and the risk of bening and malignant biliary tract disease. Cancer. 2002;95(9):1946-3.

[21]World Cancer Research Fund American Institute for Cancer Research. Food, nutrition, physical activity and the prevention of cancer: a global perspective. Washington (DC):AICR;2007.

[22]Bao Y, Michaud DS, Spiegelman D, et al. Folate Intake and Risk of Pancreatic Cancer: Pooled Analysis of Prospective Cohort Studies. J Natl Cancer Inst. 2011;103(24):1840-50.

[23] Annese V, Minervini M, Gabbrielli A, et al. ABO blood groups and cancer of the pancreas. Int J Pancreatol. 1990;6(2):81-88.

[24]Gandini S, Lowenfels AB, Jaffee EM, et al. Allergies and the risk of pancreatic cancer: a meta-analysis with review of epidemiology and biological mechanisms. Cancer Epidemiol Biomarkers Prev. 2005;14(8):1908-6.

[25]Gong Y, Zhou Q, Zhou Y, et al. Gastrectomy and risk of pancreatic cancer: systematic review and meta-analysis of observational studies. Cancer Causes Control. 2012;23(8):1279-8.

[26]Bosetti C, Lucenteforte E, Bracci PM, et al. Ulcer, gastric surgery and pancreatic cancer risk: an analysis from the International Pancreatic Cancer Case-Control Consortium (PanC4). Ann Oncol. 2013;24(11):2903-10. 